\title{
THE CIRCUMSTELLAR MEDIUM SURROUNDING ROTATING MASSIVE STARS AS GRB PRECURSORS
}

\author{
B. Pérez-Rendón ${ }^{1}$, J. Higuera ${ }^{2}$, G. García-Segura ${ }^{3}$, A. Santillán ${ }^{4}$ \\ and L. Hernández-Cervantes ${ }^{5}$
}

\begin{abstract}
Long duration Gamma Ray Bursts (LGRB) are thought to originate from massive rotating stars and the interaction of their expanding jet will be affected by the structure of their circumburst medium. In this work we use rotating stellar models of massive stars to determine the state of circumbust material in various types of progenitor scenarios and we describe how this external matter can appear in GRB observations.
\end{abstract}

\section{Introduction}

Massive stars $\left(\mathrm{M}>8 M_{\odot}\right)$ lose a considerable amount of mass in form of stellar wind before the end of their life and the stellar mass loss affects the stellar evolution itself. The stellar wind parameters change in each evolutionary stage, shaping a wide variety of structures in the surrounding gas. If the collapsar conditions are fulfilled by the star, the structure of its circumstellar medium could be revealed in the signal of the long Gamma Ray Burst (LGRB) which can occur at the end of the life of these massive stars. According to Yoon et al. (2006) the collapsar model for a LGRB requires three essential ingredients: the removal of the hydrogen envelope, a massive core and enough angular moment in it, and these conditions are achieved by rotating massive stars with low metallicities that become a Wolf-Rayet star (WR) at the end of their life. In this work we use numerical simulations to describe the circumstellar medium around massive stars that fulfill these conditions in order to study the origin of the blue-shifted absorption features observed in LGRB

\footnotetext{
1 Depto. de Investigación en Física, Universidad de Sonora, Hermosillo, Sonora, México

2 Departamento de Física, Universidad de Sonora, Hermosillo, Sonora, México

3 Instituto de Astronomía, UNAM, Ensenada, Baja California, México

4 Dirección General de Cómputo y de Tecnologías de Información y Comunicación, DGCTIC, UNAM, México City, México

${ }^{5}$ Instituto de Astronomía, UNAM, Ciudad Universitaria, México City, México
} 

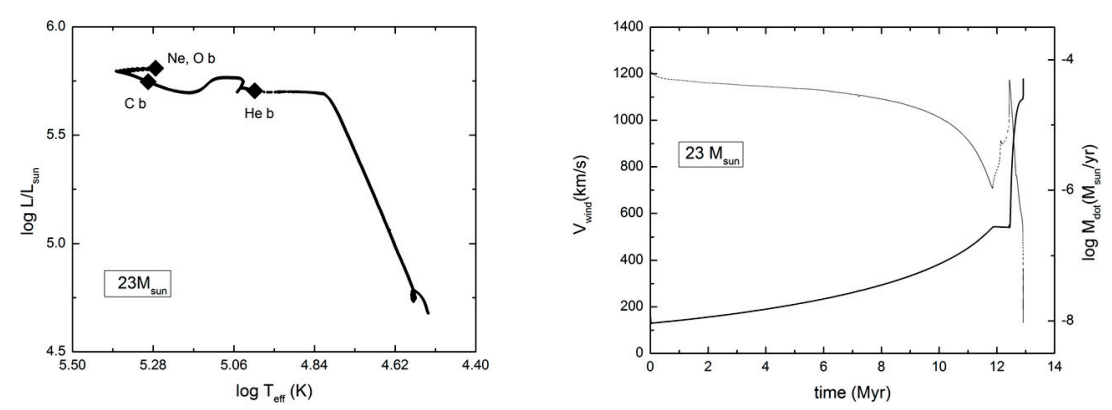

Fig. 1. Left panel: evolutionary track of a $23 M_{\odot}$ rotating star. The onset of each burning phase is indicated in the figure. Right panel: mass loss rate (thick line) and terminal velocity (broken line) of the stellar wind as function of time.

afterglows (Prochaska et al. 2008) or to explore the influence of circumstellar gas in the evolution of the LGRB itself (Ramírez-Ruiz et al. 2005). In this work we built a stellar evolution model representing a star with $\mathrm{M}_{\mathrm{ZAMS}}=23 M_{\odot}$ and we calculate their circumstellar gas evolution.

\section{Numerical simulations}

We study the case of a fast rotating $23 M_{\odot}$ star with low metallicity $\left(\mathrm{Z}=Z_{\odot} / 10\right)$. The initial angular velocity is set to $\Omega_{\mathrm{o}}=0.5 \Omega_{\text {crit }}$ where $\Omega_{\text {crit }}$ is the critical angular velocity. With this rotation rate the chemically homogeneous evolving stellar model fulfills the conditions of a stellar LGRB progenitor (as in van Marle et al. 2008). Additional to these parameters, the input physics were implemented as described in Pérez-Rendón et al. (2009). The evolutionary track in HertzpungRusell diagram (HRD) is shown in Figure 1. Due to the fast rotation this model avoids redward evolution in HRD and evolves directly to helium main sequence. We have obtained the stellar mass loss rate, wind velocity and rotation velocity as a function of time and we use it as inner boundary conditions in an explicit hydrodynamical code (ZEUS-3D, Stone \& Norman 1992) to simulate the hydrodynamical evolution of circumstellar medium, using a bidimensional grid $(400 \times 180$ cells, the longest one covering $25 \mathrm{pc}$ in the radial direction) during the entire life of the star.

\section{Results and discusion}

The medium around massive stars is continually shaped during the progenitor lifetime due the mass loss and the velocity of their winds. During main sequence (MS) the stellar wind carves a circumstellar wind blown cavity surrounding the star, bordered by a thin, dense and cold shell (Weaver et al. 1977) with a radius greater than $20 \mathrm{pc}$. At $5 \times 10^{5}$ years before the core collapse the star reaches critical rotation and the mass loss rate increases from $\log \dot{\mathrm{M}}\left(M_{\odot} \mathrm{yr}^{-1}\right)=-6.5$ to -4.3 


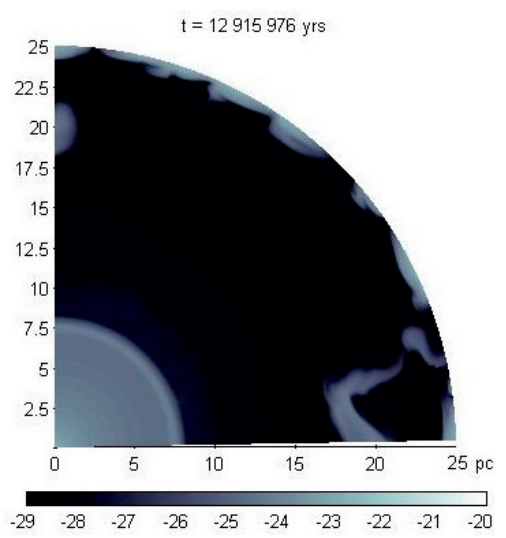

Fig. 2. Density around our $23 M_{\odot}$ rotating model at the end of its life. We show the logarithm of gas density in $\mathrm{g} \mathrm{cm}^{-3}$.

while the average wind velocity drops from $\approx 10^{3} \mathrm{~km} \mathrm{~s}^{-1}$ to small velocities about $100 \mathrm{~km} \mathrm{~s}^{-1}$. This wind is characterized by a large mass loss rate and a small wind velocity (high density wind). A shell of shocked wind starts to build up closer to the star and is unstable due to Vishniac and/or Rayleigh-Taylor instabilities while it propagates outwards.

The temporary shells in CSM created during the stellar evolution have disappeared by the time the star reaches the end of its life leaving a highly turbulent wind bubble. The last phase of slow and high density wind builds a turbulent thin shell close to the star. The explosion of the central star will then occur in this non-isotropic medium with different densities that may influence the emission of the GRB signal.

This work has been supported by CONACyT project 104651 .

\section{References}

Pérez-Rendón, B., García-Segura, G., \& Langer, N., 2009, A\&A, 506, 1249

Prochaska, J.X., Dessauges-Zavadsky, M., \& Ramírez-Ruiz, E., et al., 2008, ApJ, 685, 344

Ramírez-Ruiz, E., García-Segura, G., \& Salmonson, J.D., et al., 2005, ApJ, 631, 435

Stone, J.M., \& Norman, M.L., 1992, ApJS, 80, 753

van Marle, A.J., Langer, N., Yoon, S.-C., et al., 2008, A\&A, 478, 769

Weaver, T.A., McCray, R., Castor, J., et al., 1977, ApJ, 218, 377

Yoon, S.-C., Langer, N., \& Norman, C.A., 2006, A\&A, 460, 199 
